\title{
Бывают ли осциллографы для электромагнитного поля?
}

\author{
А. Шостак ${ }^{1}$, Д. Кондрашов², э. Сутау ${ }^{3}$, М. Крейтлоу ${ }^{4}$
}

УДК 621.317 | ВАК 05.11 .08

\begin{abstract}
Измерение уровня воздействия электрических полей на радиоэлектронную аппаратуру - актуальная задача во многих областях. Например, в процессе эксплуатации бортовое оборудование любого воздушного судна подвергается воздействию электромагнитного излучения. Это излучение обусловлено сигналами от близлежащих радиопередатчиков других устройств в частотном диапазоне от 10 кГц до 40 ГГц. Оно может привести к значительному ухудшению качества функционирования бортовых систем гражданских воздушных судов, попадающих в данную электромагнитную среду, потере работоспособности элементов и устройств, возникновению аварийных ситуаций.
\end{abstract}

ровень мощности излучения, воздействующего на бортовые системы воздушных судов, может достигать высоких значений. Например, согласно стандарту KT-160D «Условия эксплуатации и окружающей среды для бортового авиационного оборудования", уровень электрического поля при испытаниях летательных аппаратов на восприимчивость к помехам радиоизлучения достигает 7,2 кB / м (категория L). А если рассматривать требования к беспилотным летательным аппаратам (БПЛА), то на момент написания данной статьи еще нет единого стандарта, определяющего уровни воздействия на БПЛА различных типов, а также нормирование уровня излучения от них.

Проблема влияния на аппаратуру импульсного электрического поля высокой мощности стоит также и в автомобильной промышленности. Испытания на устойчивость к радиочастотному полю автомобильной электроники часто проводят в реверберационных камерах (рис. 1) (по стандартам SAE J1113 / 27, IEC 61000-4-21). Это камеры, конструкция которых позволяет полностью отражать сигналы всеми поверхностями, то есть отсутствует поглощение и нет стоячих волн, что дает возможность создавать поля напряженностью до нескольких сотен вольт на метр.

ООО "Остек-Электро», специалист технического сопровождения, Shostak.A@ostec-group.ru.

ООО "Остек-Электро", начальник группы волновых процессов, Kondrashov.D@ostec-group.ru.

Компании LUMILOOP GmbH (Германия), технический директор, eike.suthau@lumiloop.de.

4 Научно-исследовательский институт защитных технологий и защиты от ОМП Бундесвера (Мюнстер, Германия).
Для решения задач измерения импульсных электрических полей высокой мощности используют датчики напряженности электрического поля: в безэховых, реверберационных камерах и на полигонах для испытаний на устойчивость к электромагнитному полю и на помехоэмиссию. Их конструкция позволяет быстро провести измерения в различных точках испытательной площадки и оказывает минимальное влияние на полученные результаты.

В статье мы рассмотрим работу нового датчика импульсного электрического поля LSProbe 1.2 (рис. 2) немецкой фирмы LUMILOOP GmbH.

\section{УНИВЕРСАЛЬНЫЙ ДАТЧИК ПОЛЯ LSProbe 1.2}

Основное преимущество датчика - отображение значения импульсного электромагнитного поля по каждой из осей X, Y и Z с очень высокой скоростью, что обеспечивает захват очень коротких импульсов и модулированных

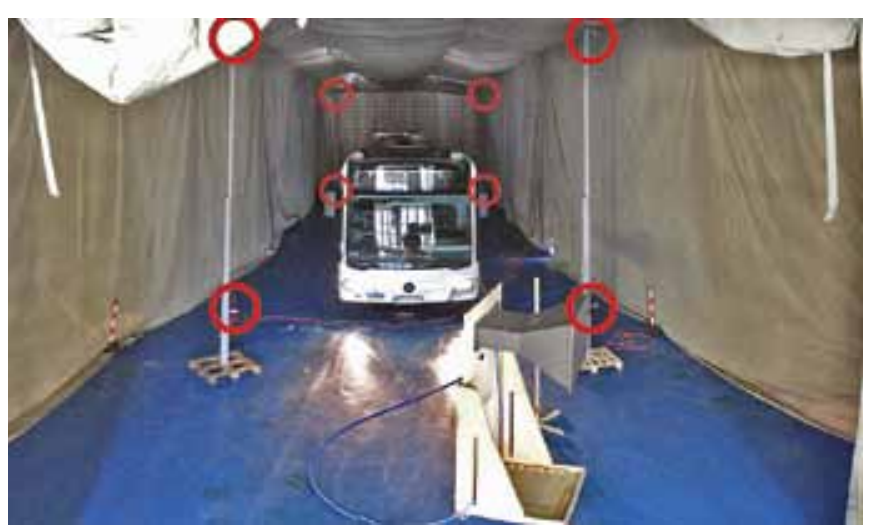

Рис. 1. Испытания автобуса в реверберационной камере. Источник: www.lumiloop.de 


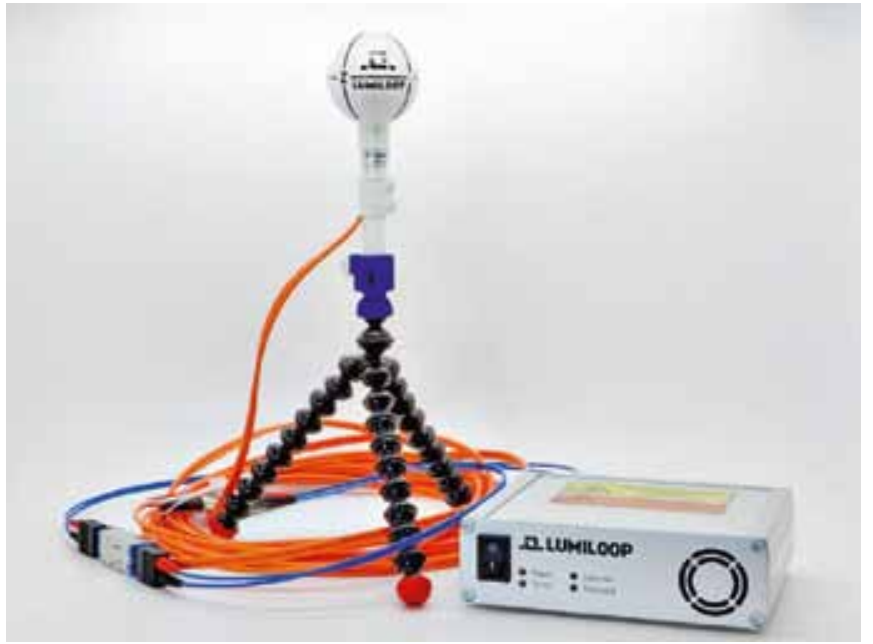

Рис. 2. Датчик импульсного электрического поля LSProbe 1.2. Источник: www.lumiloop.de

сигналов. По сути, LSProbe 1.2 является анализатором электромагнитного поля с лазерным питанием, позволяющим работать непрерывно без подзарядки или замены батареи. Для увеличения точности измерений датчик использует внутренний коэффициент калибровки и может обмениваться данными по оптоволоконным кабелям на расстояниях до 1000 м. Конструкция и калибровочные характеристики датчика обеспечивают измерение электрического поля непрерывных колебаний (CW) с напряженностью, превышающей 25 кВ/м, в диапазоне частот от 30 МГц до 12 ГГц. Однако наибольший интерес в LSProbe 1.2 представляет его функционирование при наличии сверхвысоких импульсных электромагнитных полей. Именно эти технические характеристики мы рассмотрим далее.

\section{ЭКСПЕРИМЕНТАЛЬНАЯ УСТАНОВКА}

При проведении испытаний на восприимчивость К импульсному электромагнитному полю высокой мощности

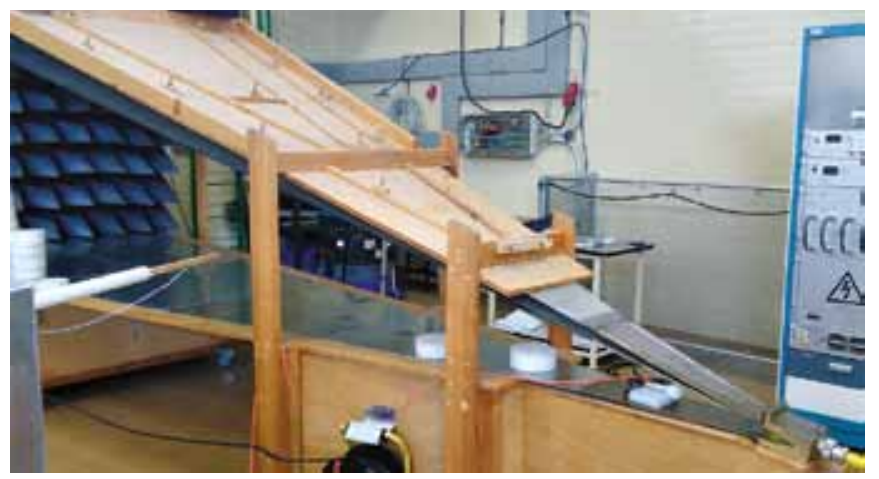

Рис. 3. Волновод СТЕМ-камеры и генератор импульсов. Источник: www.lumiloop.de датчик LSProbe 1.2 располагался по центру в боковом открытом волноводе GTEM-камеры (рис. 3). На волновод длиной около 5 м подавалось питание с генератора импульсов РВG7 компании Kentech Instruments Ltd., способного обеспечить амплитуду импульсов 45 кВ на нагрузке 50 Ом. Частота импульсов генератора составляет 500 Гц, время нарастания фронта - 150 пс, длительность -2 нс.

Измерение напряженности импульсного электромагнитного поля происходило при изменении задаваемого выходного сигнала с генератора импульсов, а также при изменении положения датчика поля внутри волновода (рис. 4). Датчик поля работал в режиме 0, который позволяет использовать диапазон частот от 30 МГц до 6 ГГц. Частота дискретизации в этом режиме составляет 500 тыс. отсчетов/с, время отклика датчика - около 330 нс

Регистрировались значения показателя уровня принимаемого сигнала (received signal strength indicator, RSSI), позволяющие получать информацию о возможном Cостоянии перегрузки датчика поля.

\section{РЕЗУЛЬТАТЫ ИСПЫТАНИЙ}

При уровнях воздействия 7, 14, 75 и 150 кВ/м испытания проводились при расположении датчика LSProbe 1.2 так, что электрическое поле было параллельно оси X датчика (рис. 4а). При уровнях 300 и 600 кВ/м датчик располагался, как показано на рис. 46, то есть электрическое поле было параллельно оси Ү датчика.

На рис. 5 показаны типичные импульсы для каждого значения напряженности поля, интервалы между импульсами были удалены программным путем для удобства отображения измеряемых значений.

На графиках видно, что значения RSSI могут быть ниже, чем уровень шума, соответствующий основной оси
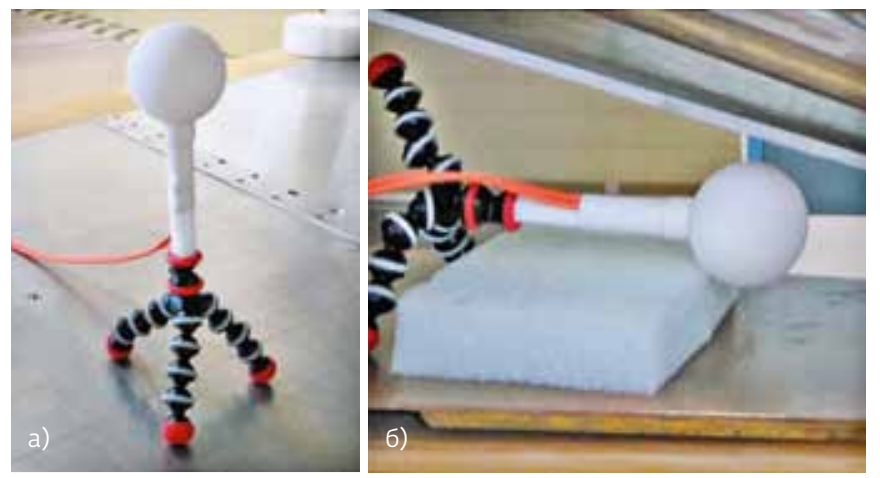

Рис. 4. Расположение датчика электрического поля: а - для измерения низких значений напряженности импульсного поля (ось $\mathrm{X} \| \overrightarrow{\mathrm{E}}$ ); 6 - для измерения высоких значений напряженности импульсного поля (ось $\mathrm{Y} \| \overrightarrow{\mathrm{E}}$ ). Источник: www.lumiloop.de 
(т.е. оси, параллельной электрическому полю). Это наблюдается при высоких уровнях сигнала (см. рис. 5д и 5е). В таких случаях можно сделать вывод, что детектор RSSI находится в состоянии перегрузки.

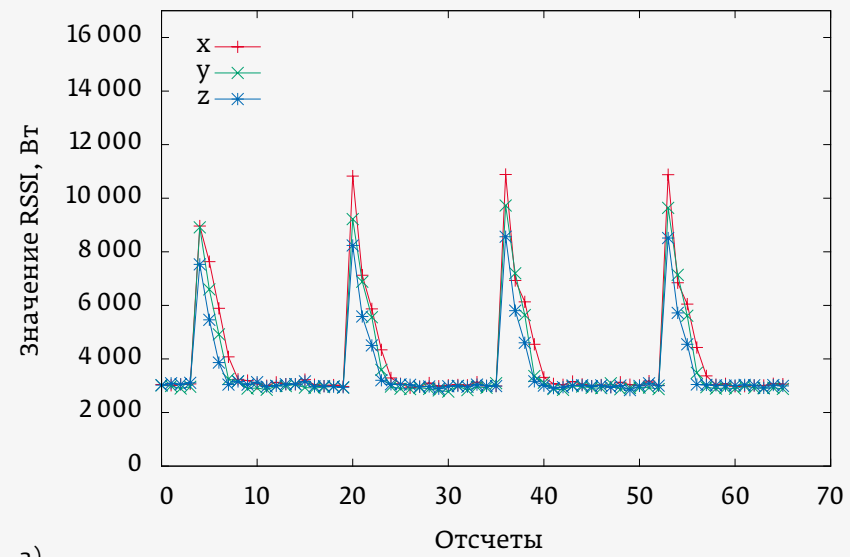

a)

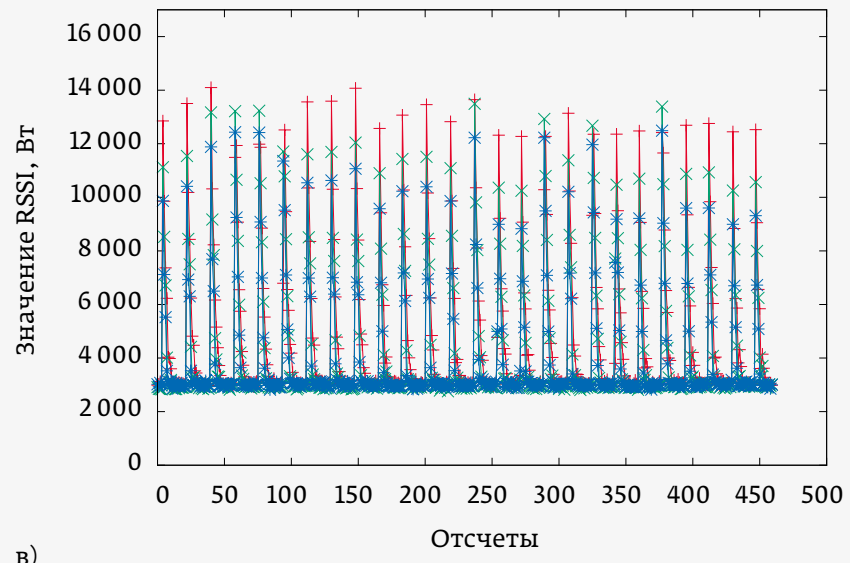

B)

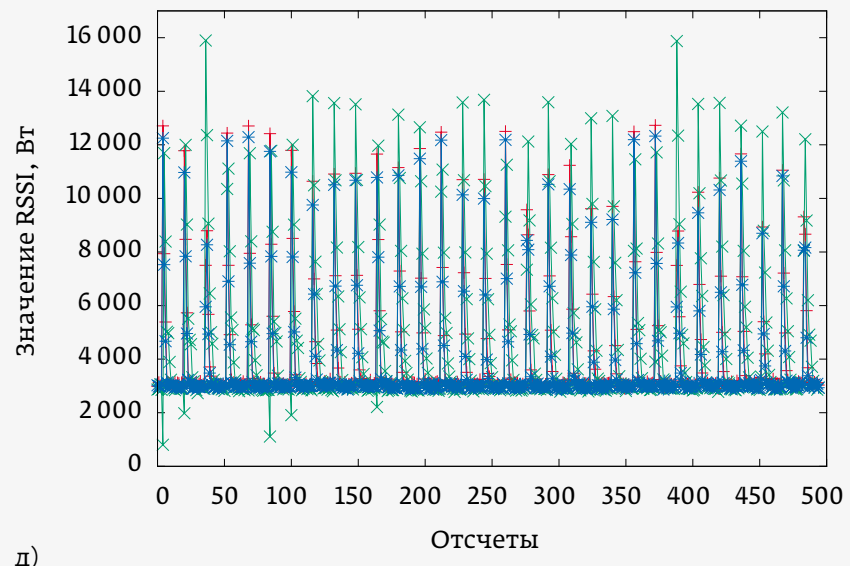

На рис. 6 представлены значения RSSI в зависимости от напряженности поля. На графике четко видны максимальные значения RSSI, которые пропорциональны напряженности поля, создаваемого импульсами генератора
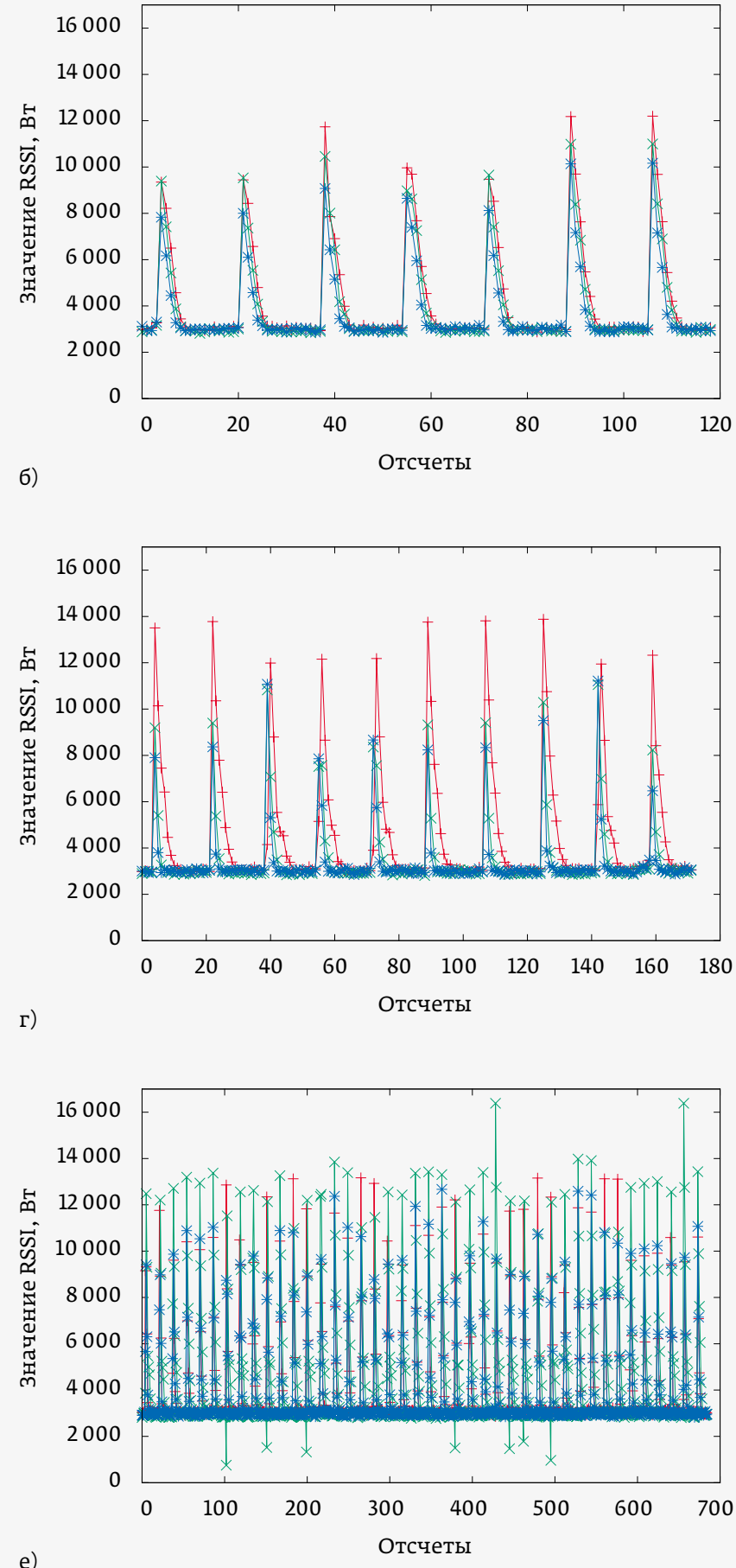

Рис. 5. Значения RSSI для различных значений напряженности импульсного поля: а - 7 кB/ в - $75 \mathrm{kB} / \mathrm{M} ;$ г $-150 \mathrm{kB} / \mathrm{M} ;$ д - $300 \mathrm{kB} / \mathrm{M} ;$ е - $600 \mathrm{kB} / \mathrm{M}$. Скорость измерений $-5 \cdot 10^{5}$ отсчетов/с. Источник: www.lumiloop.de 
Результаты для сигнала с уровнем 150 кВ/м являются небольшим исключением. По причине статистического характера измерений и их небольшого числа для данной напряженности поля реальное пиковое значение не было зафиксировано должным образом. В каждом случае значения, соответствующие осям, перпендикулярным вектору электрического поля, отличаются от значений для основной оси примерно на 20 дБ.

Таким образом, датчик импульсного электрического поля LSProbe 1.2 демонстрирует стабильную работу при значениях напряженности поля до $600 \mathrm{kB} / \mathrm{M}$. То есть LSProbe $1.2 \mathrm{Mo-}$ жет подвергаться воздействию импульсных электрических полей высокой мощности, пока их среднее значение не превышает пикового, на которое рассчитан датчик.

Можно отметить, что вход и детектор мощности датчика поля действуют как фильтр нижних частот для входных импульсов. Разумеется, датчик поля не может определить истинное максимальное значение электрического поля, поскольку время отклика датчика слишком велико. Однако можно показать, что относительная величина амплитуды импульса поля пропорциональна наблюдаемому значению напряженности поля. Поэтому датчик LSProbe 1.2 можно использовать для измерения относительной напряженности электрического поля при работе с генератором, вырабатывающим непрерывную последовательность одинаковых импульсов.

Измерения были выполнены с использованием специального аппаратного и программного обеспечения, позволяющего обрабатывать и отображать полученные данные, оно поставляется вместе с датчиком поля.

Серийная версия датчика электрического поля LSProbe 1.2 имеет ряд функций, которые значительно упрощают измерение импульсных полей:

- Режим "Выброс". Измерения выполняются со скоростью 2 млн отсчетов в секунду, позволяя увеличить разрешение по времени в четыре раза.

- запуск по уровню поля. Пиковое значение импульсного электрического поля можно легко определить, используя запуск по уровню поля и устанавливая этот уровень как можно более высоким. Так как частота дискретизации датчика поля и частота повторения импульсов не синхронизированы, то в конце концов будут получены результаты сэмплирования в области пиковых значений

импульсного поля, что позволит построить приближенный график данных пиковых значений и оценить максимальный уровень напряженности поля.

- Режим статистики. Поставляемое с датчиком программное обеспечение позволяет осуществлять непрерывный сбор статистики о напряженности поля. Режим статистики включает измерение максимальной напряженности поля и распределения напряженности поля в течение неограниченного времени. Импульсное поле с постоянной формой импульса создает характерное распределение относительной напряженности поля, которое может быть откалибровано для каждой формы импульса, что позволяет получить абсолютные значения напряженности поля.

Таким образом, датчик LSProbe 1.2 - это высокопроизводительная платформа для измерения непрерывных и импульсных электрических полей. Описанные испытания подтверждают, что LSProbe 1.2 - это своего рода "осциллограф" для электромагнитного поля, он измеряет амплитуды полей высокой напряженности и регистрирует их значения во временной области, которые отображаются благодаря сопутствующему программному обеспечению. В перспективе использование такого датчика позволит повышать уровни электромагнитных воздействий для проверки устойчивости радиоэлектронной аппаратуры, например, устройств авиакосмической отрасли с верхней границей частотного диапазона до 18 или 40 гГц. 